\title{
ESTUDO DA ATIVIDADE CATALÍTICA DO PÓ DE OSSO BOVINO IMPREGNADO COM Fe(III): NOVO MATERIAL PARA FOTODEGRADAÇÃO DO CORANTE ÍNDIGO CARMIM
}

\author{
M. G. COELHO ${ }^{1}$, L. O. $\operatorname{SILVA}^{3}$, R. AUGUSTI ${ }^{1}$, G. M. DE LIMA ${ }^{1}$, J. D. ARDISSON ${ }^{2}$ e F. V. \\ DE ANDRADE ${ }^{1}$ \\ ${ }^{1}$ Universidade Federal de Minas Gerais, Departamento de Química \\ ${ }^{2}$ Centro de Desenvolvimento em Tecnologia Nuclear \\ ${ }^{3}$ Centro Universitário Newton Paiva, Instituto de Ciências Exatas \\ E-mail para contato: proquimarcio@gmail.com
}

\begin{abstract}
Um novo material para fotodegradação do corante índigo carmim é proposto neste trabalho. O material é constituído de pó de osso bovino impregnado com $\mathrm{Fe}(\mathrm{III})$, seu desempenho como fotocatalisador em meio aquoso foi avaliado. O material foi caracterizado por difração de raios-X de pó (DRX), espectroscopia Mössbauer de ${ }^{57} \mathrm{Fe}$, medida de área superficial (BET) e espectrometria de absorção atômica. O processo de degradação foi monitorado por espectroscopia de ultravioleta na região do visível (UV-Vis) e os subprodutos caracterizadas por ESI(-)MS (espectrometria de massas com fonte de ionização por eletrospray no modo negativo). O fotocatalisador foi capaz de causar a descoloração completa de soluções de índigo com luz solar ou ultravioleta. Um mecanismo para a degradação foi proposto.
\end{abstract}

\section{INTRODUÇÃO}

De acordo com testes realizados pela Associação Ecológica e Toxicológica da Indústria de Transformação de Corantes, mais de $90 \%$ dos 4.000 corantes normalmente utilizados em processos industriais apresentam algum nível de toxicidade (Nigam et al., 2000; Robinson et al., 2001; Golka et al., 2003). Seus efluentes industriais são motivo de preocupação, por causa dos efeitos toxicológicos e ambientais sobre uma série de microorganismos. Quantidades muito pequenas de corantes podem ser facilmente reconhecidas tanto em produtos industriais ou em seus resíduos líquidos (Robinson et al., 2002). Os principais problemas estão relacionados com importantes propriedades dos corantes: a estabilidade química e a estabilidade térmica. Embora necessárias para aplicações industriais, estas propriedades dificultam o tratamento dos resíduos aquosos, mesmo em baixas concentrações.

A degradação de substratos orgânicos em solução aquosa tem sido geralmente promovida por processos oxidativos avançados (POAs). Nos POAs, os radicais hidroxila oxidantes extremamente reativos e poderosos, gerados in situ, promovem a oxidação da molécula alvo. Os POAs compreendem combinações de radiação UV e $\mathrm{H}_{2} \mathrm{O}_{2}, \mathrm{TiO}_{2}, \mathrm{O}_{3}$, radiação ultra-som, e o reagente Fenton (Lee et al., 2006). Um grande número de artigos descrevem a aplicação dos 
POAs para degradar corantes em solução aquosa. Por exemplo, uma matriz composta por $\mathrm{TiO}_{2}$ imobilizada nas superfícies internas de tubos de quartzo expostos continuamente a uma fonte de UV tem sido utilizada com sucesso para degradar vários corantes (Urzedo et al., 2007). Experiências em pequena escala mostraram que o sistema $\mathrm{TiO}_{2} / \mathrm{luz}$ solar possui alta eficiência na remoção da cor a partir de soluções aquosas de indigo carmim (Liao et al., 2009). Os estudos sobre a degradação de corantes em soluções aquosas normalmente têm-se centrado na eficiência descoloração promovida pelos POAs, enquanto que tem sido dada pouca atenção para a caracterização de sub-produtos formados. Uma técnica alternativa para ser usada para este fim é a espectrometria de massas com fonte de ionização por eletrospray (ESI-MS). Esta técnica possui a capacidade notável de transferir suavemente espécies da fase condensada para a fase gasosa sem induzir reações indesejáveis. Por causa disso, ESI-MS pode ser aplicada com sucesso no monitoramento de metodologias ambientais (Coelho et al., 2010).

O objetivo do presente trabalho foi preparar e caracterizar um novo material formado pela impregnação de pó de osso bovino com Fe(III). Uma das características vantajosas deste novo material é que é barato e simples de preparar. O suporte, osso bovino, é um resíduo final da produção de alimentos. A capacidade do material em atuar como um fotocatalisador da degradação do corante têxtil indigo carmim, em meio aquoso, foi avaliada. A espectrometria de massas com fonte de ionização por eletrospray (ESI-MS) foi utilizada para caracterizar os subprodutos resultantes do processo de degradação.

\section{PARTE EXPERIMENTAL}

\subsection{Obtenção do Fotocatalisador}

Um pedaço seco de osso bovino foi lavado e triturado. Uma quantidade equivalente a $1 \mathrm{~g}$ do pó do osso bovino foi imersa em uma solução aquosa de $\mathrm{FeCl}_{3}\left(1 \mathrm{~mol} \mathrm{~L}^{-1}\right)$. Depois de $24 \mathrm{~h}$ de agitação, o sólido marrom foi tratado termicamente na temperatura de $200{ }^{\circ} \mathrm{C}$ por $1 \mathrm{~h}$. O material obtido foi caracterizado por difração de raios-X, espectroscopia Mössbauer de ${ }^{57} \mathrm{Fe}$, área superficial BET e espectrometria de absorção atômica.

\subsection{Testes fotocatalíticos}

O material preparado $(30 \mathrm{mg}$ ) foi adicionado a uma solução aquosa do índigo carmim (100 mL; $\left.30 \mu \mathrm{mol} \mathrm{L} \mathrm{L}^{-1}\right)$ para a realização dos experimentos fotocatalíticos. Depois da adição do catalisador o sistema foi exposto à luz ultravioleta ou luz solar. Alíquotas foram retiradas durante o experimento e analisadas por espectrofotometria de UV/Vis e espectrometria de massas com fonte de ionização por eletrospray (ESI-MS). 


\section{RESULTADOS E DISCUSSÕES}

\subsection{Caracterização do material}

O pó de osso bovino impregnado apresentou uma área superficial de $4 \mathrm{~m}^{2} \mathrm{~g}^{-1}$. A absorção atômica revelou que a quantidade de ferro no material preparado é de $5 \%$ em massa. O difratograma de raios- $\mathrm{X}$ mostrou que o material é completamente amorfo, uma vez que não foram observadas linhas de difração. Já nos experimentos de Mössbauer ${ }^{57} \mathrm{Fe}$ (Figura 1) foi possível identificar duas fases de ferro no material: $\mathrm{Fe}(\mathrm{III}), 83 \%$ (desvio isomérico, $\delta=0.36 \mathrm{~mm} \mathrm{~s}^{-1}$ e desdobramento quadrupolar, QS $\left.=0.89 \mathrm{~mm} \mathrm{~s}^{-1}\right)$ e Fe(II), $17 \%\left(\delta=1.12 \mathrm{~mm} \mathrm{~s}^{-1}\right.$ e $\mathrm{QS}=2.25 \mathrm{~mm} \mathrm{~s}^{-1}$ ).

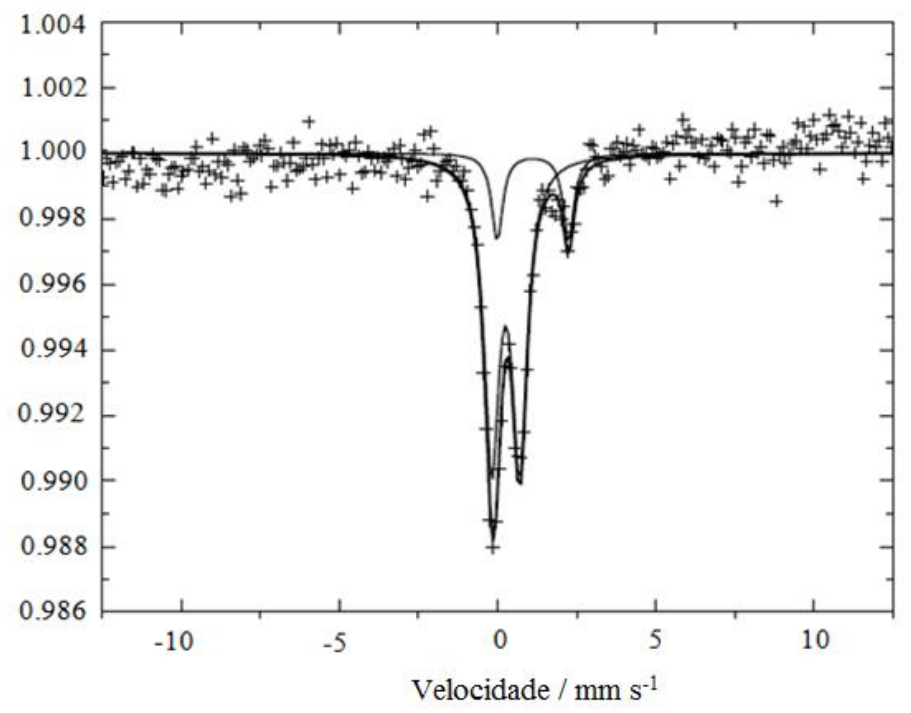

Figura 1.Espectro de Mössbauer de ${ }^{57} \mathrm{Fe}$ do material Fe(III)/pó de osso bovino.

\subsection{Testes Fotocatalíticos}

A solução aquosa contendo o corante índigo carmim foi completamente descolorida em 60 min de reação no sistema contendo o catalisador preparado e luz UV (Figura 2). A velocidade de degradação da molécula do corante é bem menor quando se utiliza luz solar ao invés de luz UV. Para efeito de comparação outros testes foram realizados como a utilização de osso/UV, somente osso/sem luz, somente UV, e ferro lixiviado do catalisador. O catalisador preparado na presença de luz UV foi o mais eficiente na remoção do corante da solução. 


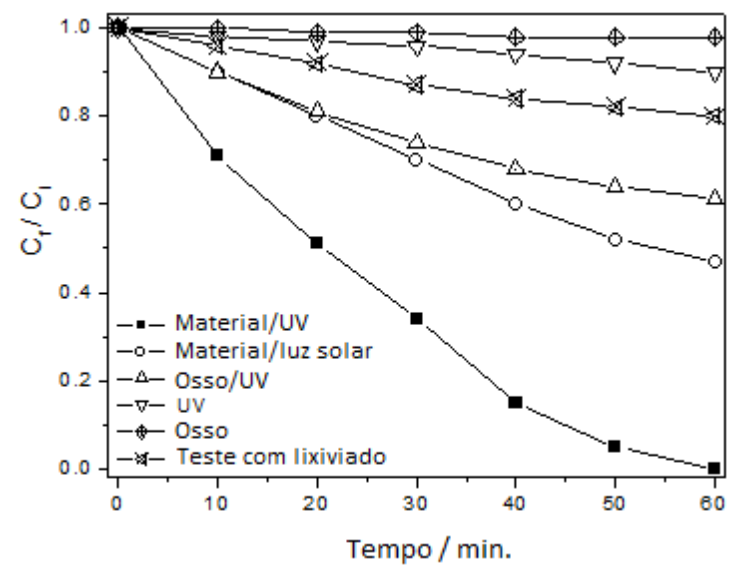

Figura 2: Monitoramento da degradação do corante índigo carmim em solução aquosa usando luz solar e luz ultravioleta.

A espectrometria de massas com fonte de ionização por eletrospray (ESI-MS), no modo negativo, foi utilizada com o objetivo de monitorar a degradação da molécula do corante. A presença do ânion de m/z 421 é relativa à molécula do índigo desprotonada, [1 - H], na solução inicial do corante (Figura 3a). No espectro de massas da solução depois de decorrida a reação, Figura 3b, o ânion de m/z 421 não é detectado, indicando que a molécula do corante foi totalmente consumida, ânions de m/z 226 (o mais abundante), 244 e 216 são observados claramente.

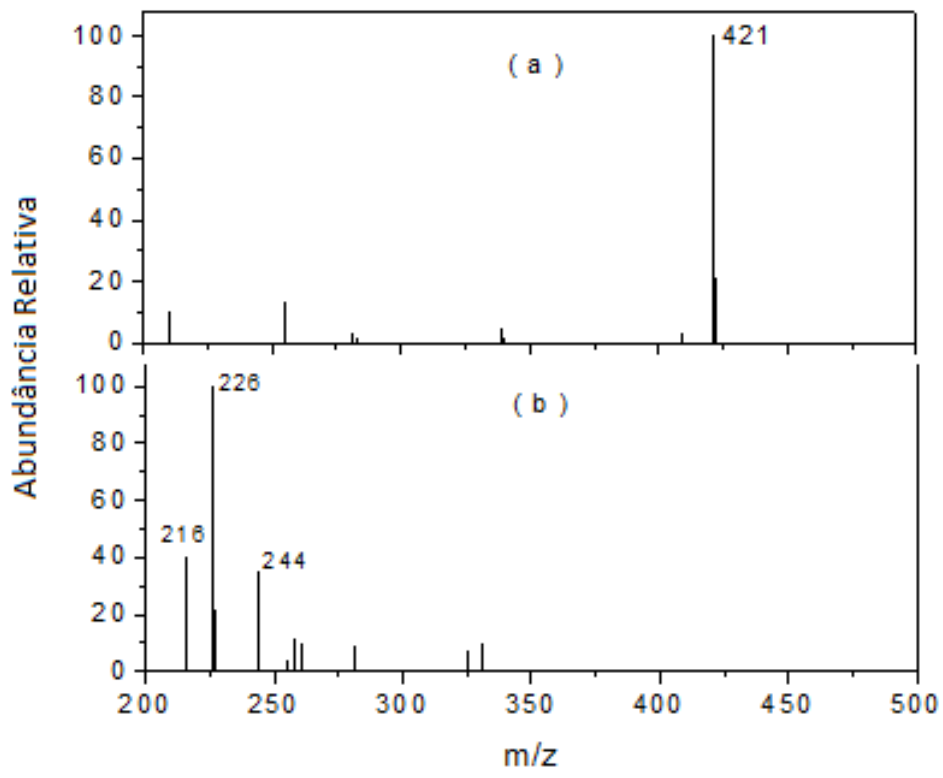

Figura 3: Espectro de massas da solução aquosa do corante índigo carmim: (a) antes do processo fotocatalítico e (b) após 60 min de reação. 
Com base nos resultados de espectrometria de massas foi possível propor um mecanismo de reação para o processo fotocatalítico (Figura 4). O produto (2), com uma massa nominal de 227 foi detectado com m/z 226 (desprotonado [2 - H]), é formado a partir da oxidação do corante (1). Tal processo oxidativo compreende essencialmente uma segmentação da ligação $\mathrm{C}=\mathrm{C}$ exocíclica seguida pela inserção de átomos de oxigênio nas posições C2 e C2 ' de (1) (Figura 4) e ocorre provavelmente na superfície do compósito. A formação constante de (2), que não tem nenhum sistema conjugado como em (1), poderia assim explicar a descoloração contínua da solução, como observado no experimento. A formação dos outros produtos [(3) e (4)] a partir do composto (2) poderia facilmente ser explicada tomando em consideração alguns caminhos de reação simples e lógicos, como indicados no esquema 1 . Tais produtos foram detectados como $[3-\mathrm{H}]^{-}$de m/z 244 e $[4-\mathrm{H}]^{-}$de m/z 216, segundo as indicações da Figura 3b.

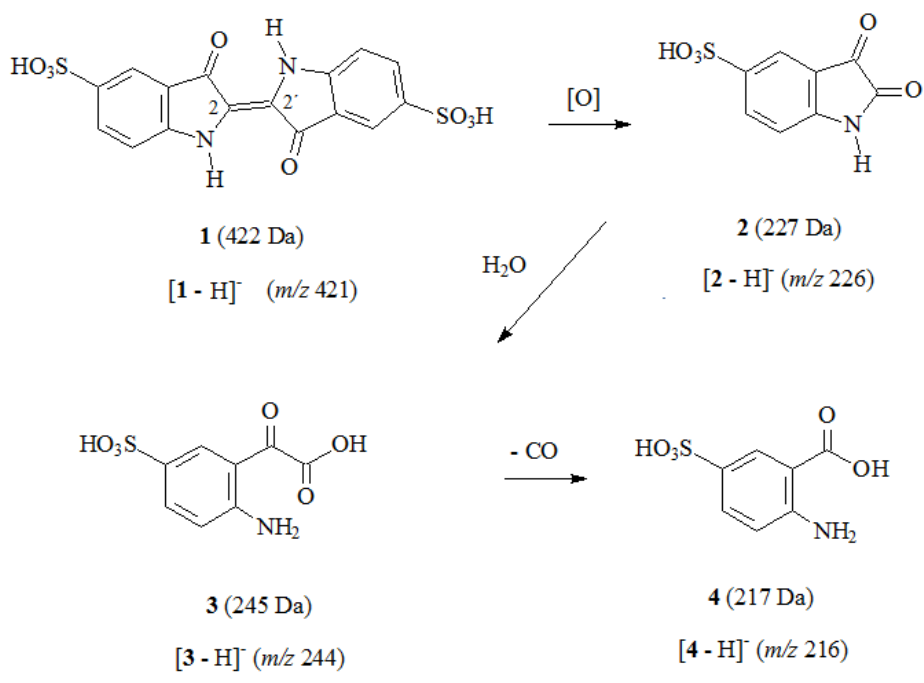

Figura 4: Proposta de mecanismo de reação para a degradação da molécula do corante índigo carmim catalisada pelo material preparado.

\section{CONCLUSÕES}

O material preparado foi utilizado como um catalisador no processo fotocatalítico. O sistema foi capaz de causar a descoloração completa de soluções de índigo carmim.

De acordo com os dados obtidos por ESI-MS, um mecanismo para a degradação das moléculas de índigo carmim foi proposto. Independentemente da fonte de luz (solar ou ultravioleta), os mesmos tipos de produtos foram formados, indicando assim que o mecanismo 
envolvido é provavelmente o mesmo. Este é um resultado consistente, uma vez que ambos os processos prosseguem através da geração de radicais hidroxila, estes promovem a degradação das moléculas do corante.

\section{REFERÊNCIAS}

COELHO, M. G.; de LIMA, G. M.; AUGUSTI, R.; MARIA, R. D. A.; ARDISSON, J. D., New materials for photocatalytic degradation of Indigo Carmine Synthesis, characterization and catalytic experiments of nanometric tin dioxide-based composites. Appl. Catal. B, v. 96, p. $67-71,2010$.

GOLKA, K.; KOPPS, S.; MYSLAK, Z. W., Carcinogenicity of azo colorants: influence of solubility and bioavailability.Toxicol. Lett., v. 151, p. 203 - 210, 2004.

LEE, J. W.; CHOI, S. P.; THIRUVENKATACHARI, R.; SHIM, W. G.; MOON, H., Evaluation of the performance of adsorption and coagulation processes for the maximum removal of reactive dyes, Dyes Pigm., v. 69, 196 - 203, 2006.

NIGAM, P.; ARMOUR, G.; BANAT, I. M.; SINGH, D.; MARCHANT, R.; MCHALE, A. P.; MCMULLAN, G., Physical removal of textile dyes from effluents and solid-state fermentation of dye-adsorbed agricultural residues.Bioresour. Technol.,v.72, p. 219 - 226, 2000.

ROBINSON, T.; MCMULlAN, G.; MARCHANT, R.; NIGAM, P., Remediation of dyes in textile effluent: a critical review on current treatment technologies with a proposed alternative. Bioresour. Technol., v. 77, 247 - 255, 2001.

ROBINSON, T.; CHANDRAN, B.; NIGAM, P., Removal of dyes from an artificial textile dye effluent by two agricultural waste residues, corncob and barley husk. Environ. Int., v. 28, p. $29-33,2002$.

URZEDO, A. P. F. M.; NASCENTES, C. C.; DINIZ, M. E. R.; CATHARINO, R. R.; EBERLIN, M. N.; AUGUSTI, R., Indigo Carmine degradation by hypochlorite in aqueous medium monitored by electrospray ionization mass spectrometry, Rapid Comm. Mass Spectrom., v. 21, 1893 - 1899, 2007. 
Liao, H.; Stenman, D.; Jonsson, M., Study of Indigo carmine as radical probe in photocatalysis, $J$.

Photochem. Photobiol., A, v. 202, p. 86 - 91, 2009. 